\title{
Supersoft Elasticity in Polydomain Nematic Elastomers
}

\author{
J. S. Biggins and M. Warner \\ Cavendish Laboratory, University of Cambridge, Cambridge, CB3 OHE, United Kingdom \\ K. Bhattacharya \\ Division of Engineering and Applied Science, California Institute of Technology, Pasadena, California 91125, USA
}

(Received 22 April 2009; published 15 July 2009)

\begin{abstract}
We consider the equilibrium stress-strain behavior of polydomain liquid crystal elastomers (PLCEs). We show that there is a fundamental difference between PLCEs cross-linked in the high temperature isotropic and low temperature aligned states. PLCEs cross-linked in the isotropic state then cooled to an aligned state will exhibit extremely soft elasticity (confirmed by recent experiments) and ordered director patterns characteristic of textured deformations. PLCEs cross-linked in the aligned state will be mechanically much harder and characterized by disclination textures.
\end{abstract}

DOI: 10.1103/PhysRevLett.103.037802

Liquid crystal elastomers are rubbery materials that possess liquid crystal order [1]. Their large spontaneous elongations at the symmetry-breaking isotropic-nematic transition have long made them ideal candidates for showing soft (zero energy) elastic modes [2,3] in the manner proposed by Golubovic and Lubensky [4]. Monodomain samples show qualitatively soft elasticity over a large range of deformations $[5,6]$. However, monodomains never show perfectly soft behavior because, to achieve their macroscopic alignment of the director, they require the imprinting of a direction on the network, breaking the isotropy of the high temperature state. A microscopic region extracted from a polydomain cross-linked in either the nematic or the isotropic state would have fairly soft elastic modes. As anticipated by Golubovic and Lubensky, in the isotropic cross-linking case these modes will be almost perfectly soft, while in the nematic cross-linking case they will only be qualitatively soft like those in monodomains. However, the macroscopic softness of the polydomain sample is not guaranteed because the soft modes of neighboring domains may not be elastically compatible, although there are experimental suggestions of softness [7]. Using textured deformations introduced by DeSimone and Dolzmann [8] we show that polydomains cross-linked in the isotropic state retain their extreme softness macroscopically, while polydomains cross-linked in the nematic state will not. This distinction has been confirmed by recent experiments [9].

Polydomains cross-linked in the isotropic state can only deviate from perfect Golubovic-Lubensky soft elasticity because local fluctuations in, say, cross-link orientation, compromise the isotropy of the cross-linking state. Following [10] we model these fluctuations using a random field which drives the formation of the polydomain state and limits the softness of the macroscopic response.

The distinctive behavior of monodomain samples has been well understood using a neoclassical model of Gaussian distributed chains $[1,11]$. The chain conforma-
PACS numbers: 61.30.Vx, 81.40.Jj, 83.80.Va

tions are biased by the liquid crystal order so that the second moment of the conformation distribution of a free chain is $\left\langle R_{i} R_{j}\right\rangle \propto \underline{\underline{\ell}}=r^{-1 / 3}(\underline{\underline{\delta}}+(r-1) \hat{\mathbf{n}} \hat{\mathbf{n}})$, where $\mathbf{R}$ is the polymer span vector, $\underline{\underline{\delta}}$ is the identity matrix, $\hat{\mathbf{n}}$ is the nematic director, and $r$ is the anisotropy of the polymer conformation distribution which measures the coupling between the polymers and the director. If an elastomer is cross-linked in a state with scaled step-length tensor $\underline{\underline{\ell}}_{0}=$ $r^{-1 / 3}\left(\underline{\underline{\delta}}+(r-1) \hat{\mathbf{n}}_{0} \hat{\mathbf{n}}_{0}\right)$ and then subject to a deformation gradient $\underline{\underline{\gamma}}$ and a change in the step-length tensor to $\underline{\underline{\ell}}$, the free energy density is

$$
F=\frac{1}{2} \mu \operatorname{Tr}\left(\underline{\underline{\gamma}} \cdot \underline{\underline{\ell}} \underline{\underline{\underline{\gamma}}} \cdot \underline{\underline{\gamma}}^{T} \cdot \underline{\underline{\ell}}-\underline{\underline{\gamma}} \cdot\left(\underline{\underline{\delta}}-\hat{\mathbf{n}}_{0} \hat{\mathbf{n}}_{0}\right) \cdot \underline{\underline{\gamma}}^{T} \cdot \hat{\mathbf{n}} \hat{\mathbf{n}}\right)
$$

The constant shear modulus $\mu$ sets the scale of this energy, and $\underline{\underline{\ell}}$ may differ from $\underline{\underline{\ell}}_{0}$ because the strength of the nematic order has changed, leading to a change in $r$, or because the nematic director has rotated to $\hat{\mathbf{n}}$. The first term in this energy is the ideal term that allows perfectly soft elasticity-deformations of the form $\underline{\underline{\gamma}}=\underline{\underline{\ell}}^{1 / 2} \cdot \underline{\underline{\ell}}^{-1 / 2}$ do not cost energy — and the second, proportional to the coefficient of nonideality, $\alpha$, causes these deformations to cost some energy by favoring alignment of the director along $\hat{\mathbf{n}}_{0}$. This form, first derived microscopically [12] is quite generic [13] and describes the elasticity of monodomain elastomers very well $[5,6]$.

The individual domains (regions of homogenous nematic director $\hat{\mathbf{n}}$ ) in polydomain elastomers are very much larger than any of the microscopic length scales [14], so we model nematic polydomains by assuming that each domain has the same free energy as a monodomain. Therefore we can easily write down the energy functions for polydomain elastomers by simply allowing the quantities $\hat{\mathbf{n}}, \hat{\mathbf{n}}_{0}$, and $\underline{\underline{\gamma}}$ to become spatially varying fields. We distinguish between four types of polydomain, ideal and nonideal samples with nematic and isotopic cross-linking 
states, giving us four energy functions,

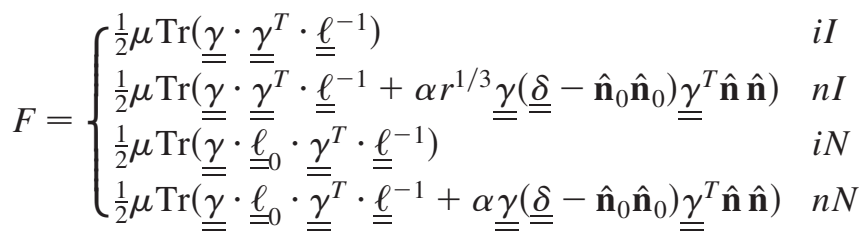

where $i$ and $n$ denote ideal and nonideal, $I$ and $N$ denote isotropic and nematic cross-linking and, in the isotropic cross-linking case the step-length tensor at cross-linking, $\underline{\underline{\ell_{0}}}$, is simply identity. In each case $\underline{\underline{\gamma}}$ is the deformation gradient from the cross-linking state.

In the nematic cross-linking case $\underline{\underline{\gamma}}=\underline{\underline{\delta}}$ minimizes the energy, and the $\hat{\mathbf{n}}_{0}(\mathbf{x})$ field is literally the nematic director field at cross-linking. The isotropic cross-linking energies are very different. Their local energy is minimized by a spontaneous elongation by a factor of $r^{1 / 3}$, which would be the shape change associated with the phase change from the isotropic cross-linking state to a monodomain nematic state. Monodomains can be synthesized with this factor lying anywhere between 5\% and 400\% [15], implying values for $r$ between 1.1 and 60 . In the ideal case this elongation can be in any direction. Since the cross-linking state is truly isotropic (unlike even isotropic genesis monodomains, which have their isotropy broken by an imposed stress at cross-linking) we expect the ideal model, which has no $\hat{\mathbf{n}}_{0}$ dependence, to be very good. However, there are extremely weak mechanisms that can introduce a locally preferred direction into the apparently isotropic state, for example, the cross-linking molecules are rod shaped and, in any given region, because there is a finite number of rods, there will be a slight average orientation $[14,16]$. This permits the inclusion of a very small nonideal term in even the isotropic cross-linking case. However, it will certainly be very much weaker than in monodomain networks, which have a preferred direction imprinted on them, or nematic cross-linking polydomains, which can distinguish the nematic director at cross-linking so that, for example, the cross-linking rods can all align in this direction.

We wish to study the macroscopic elasticity of large blocks of polydomain elastomers, which is to say we want to find the energy of a macroscopic block occupying a region $\Omega$ after imposing a homogenous deformation gradient $\underline{\underline{\lambda}}$ to the surface $\delta \Omega$ and allowing the internal deformations $\underline{\underline{\gamma}}=\nabla \mathbf{y}$ and director patterns $\hat{\mathbf{n}}(\mathbf{x})$ to relax to their optimal configurations. We define this relaxed energy function as

$$
F^{r}\left(\underline{\underline{\lambda}}, \hat{\mathbf{n}}_{0}(\mathbf{x})\right)=\min _{\substack{\mathbf{y}=\lambda . \mathbf{x} \\ \text { on } \bar{\delta} \Omega}} \min _{\hat{\mathbf{n}}(\mathbf{x})} \frac{1}{\operatorname{Vol} \Omega} \int_{\Omega} F\left(\nabla \mathbf{y}, \mathbf{n}(\mathbf{x}), \mathbf{n}_{0}(\mathbf{x})\right) d \mathbf{x} .
$$

The four different types of polydomains correspond to the different choices of $F$ in Eq. (2). All deformations in elastomers occur at constant volume, so we also require that $\operatorname{det} \nabla \mathbf{y}=1$. We will adopt the convention that $\gamma(\mathbf{x})=$ $\nabla \mathbf{y}$ is the local deformation and $\underline{\underline{\lambda}}=\langle\underline{\underline{\gamma}}\rangle$ is the macroscopic deformation.

The energy function for ideal isotropic genesis ( $i I)$ polydomains has no spatial variation, indeed it is exactly the same energy that is used to model ideal nematic monodomains. It is locally minimized by any deformation gradient of the form $\underline{\underline{\gamma}}=\nabla \mathbf{y}=\underline{\underline{\ell}}^{1 / 2}$, a uniaxial extension along $\hat{\mathbf{n}}$, which can point in any direction. A macroscopically homogenous deformation will be relaxed if it can be made out of a pattern of deformations that are all uniaxial extensions by $r^{1 / 3}$ but which average to the macroscopic deformation. Trivially, if every point in the material undergoes the same uniaxial extension by $r^{1 / 3}$ this condition is satisfied, and a relaxed aligned monodomain is formed with the director along the axis of extension. This means that stretching the cross-linking state by $r^{1 / 3}$ in any direction yields a relaxed low energy state, and there are soft deformations that map between these states. Much less obviously, DeSimone and Dolzmann showed that there are also very special deformation patterns (textured deformations-see Fig. 1) that allow a macroscopic $\underline{\underline{\lambda}}=$ $\operatorname{diag}(\lambda, 1 / \sqrt{\lambda}, 1 / \sqrt{\lambda})$, imposed in any direction, to be built entirely out of uniaxial stretches of $r^{1 / 3}$ for all $\lambda \leq r^{1 / 3}$ and hence imposed softly [8]. For $\lambda \geq r^{1 / 3}$ the energy is not soft and is minimized by the local deformation simply following the macroscopic deformation. This remarkable result means that an ideal isotropic genesis polydomain

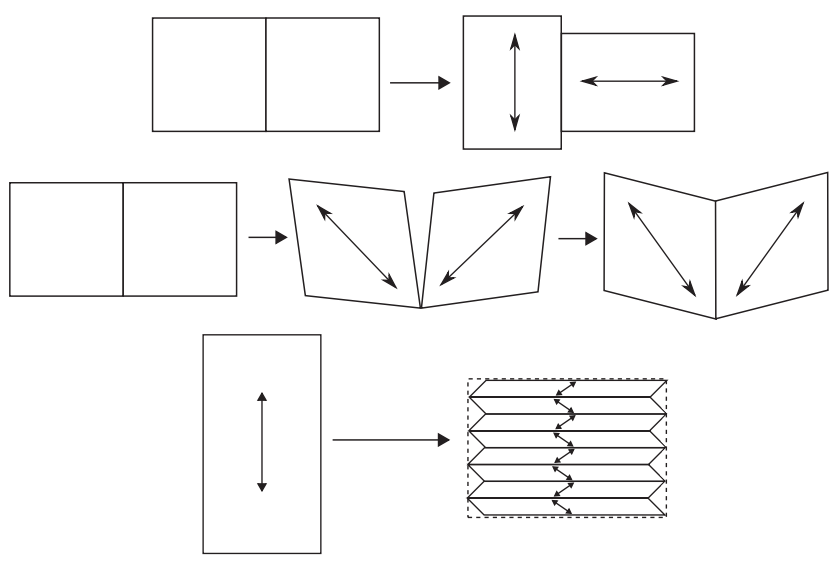

FIG. 1. Upper: Uniaxial stretches applied in different directions in different regions generally result in fracture. Middle: If the axes are bisected by the interfacial plane, body rotations can restore material continuity. Many repetitions of this type of structure make a textured deformation. Bottom: A familiar example of a textured deformation-the stripe domain seen in nematic monodomains [6]. A nematic monodomain stretched perpendicular to its director adopts a laminate structure with all laminates being stretched but also having alternating director rotation and shear which make the deformations soft. The sample has no macroscopic shear but has sheared (and hence deformed softly) at every point. 
that is stretched uniaxially should deform at zero stress $\left(F=\frac{3}{2} \mu\right)$ for $\lambda \leq r^{1 / 3}$ and then behave as a simple Hookean solid $\left(F=\frac{1}{2} \mu r^{1 / 3}\left(\lambda^{2} / r+2 / \lambda\right)\right)$ for $\lambda \geq r^{1 / 3}$,

$$
\sigma(\lambda)=\frac{d F}{d \lambda}= \begin{cases}0 & \lambda \leq r^{1 / 3} \\ \mu r^{1 / 3}\left(\lambda / r-1 / \lambda^{2}\right) & \lambda \geq r^{1 / 3}\end{cases}
$$

This macroscopic soft elasticity has a very different form to that seen in monodomains where the soft modes have a two dimensional form $(\underline{\lambda}=\operatorname{diag}(\lambda, 1,1 / \lambda))$ and deformations are soft up to $\lambda=\sqrt{r}$ for stretching perpendicular to the director [6], but never soft if the stretch is parallel to the director. All the domains in a relaxed isotropic genesis polydomain are individually able to stretch in this soft two-dimensional way, without fracturing the material, until complete alignment of the sample is reached. This is possible because the transition to the nematic happens after cross-linking, restricting the choice of domain patterns to those with this property.

We compare the above ideal stress-strain relation (3) with some recent observations [9] of an isotropic genesis polydomain rubber in Fig. 2. The observations and the predictions agree very well, clearly confirming that isotropic genesis polydomains exhibit very soft elasticity. However, the real elastomer does not quite deform at zero stress motivating the consideration of nonideal theories. Since the cross-linking state is almost isotropic, nonideality must be very small so the key features of the ideal model will persist. Extension will not occur at zero stress, but extensions up to $\lambda \leq r^{1 / 3}$ will occur at energies $O(\alpha) \ll 1$. The deformation patterns and director patterns must still be very close to those in the ideal case, so the observed patterns will still be characterized as textured deformations driven by elastic compatibility-typically oscillations between regions of constant deformation separated by sharp boundaries $[6,8,17]$ —not disclination textures which are not elastically compatible [18], although the choice of microstructure will change from point to point in the elastomer.

A (Taylor-like) upper bound on the relaxed nonideal isotropic genesis polydomain energy can be found by

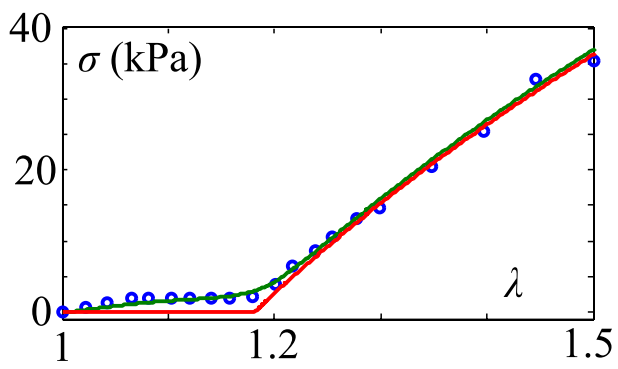

FIG. 2 (color online). Nominal stress $(\sigma)$ vs strain $(\lambda)$ for a real isotropic genesis polydomain elastomer (circles) compared with the ideal stress (lower line) and a nonideal estimate of the stress (upper line) provided by the derivative of the Sachs bound on the energy. The fitting parameters are $r=1.65, \mu=33000$ and, for the nonideal curve, $\alpha=0.01$. taking a test strain field from the ideal case and calculating its energy in the nonideal case. Since the relaxed energy function is a minimum over all strain fields, evaluating the energy at one example of a strain field is an upper bound on the energy. We take strain fields that do not correlate with the local preferred direction, so the bound can be visualized as finding the energy of ideally soft textured deformations (e.g., the bottom right diagram in Fig. 1, although the actual textures required are more complicated than these simple laminates [8]) in the presence of a randomly distributed preferred director $\hat{\mathbf{n}}_{0}$. The textured deformations in the ideal case are composed of multiple uniaxial stretches of the same magnitude. If a point in the nonideal elastomer undergoes a (local) uniaxial extension of magnitude $\gamma$ from the cross-linking state, at an angle $\theta$ to $\hat{\mathbf{n}}_{0}$, then, after minimizing over $\hat{\mathbf{n}}$ (by taking $\hat{\mathbf{n}}$ along the axis of $\underline{\underline{\gamma}}$ ) the energy of the deformation [Eq. (2) $n I]$ is

$$
F=\frac{1}{2} \mu r^{1 / 3}\left(\frac{2}{\gamma}+\left(\frac{1}{r}+\alpha\right) \gamma^{2}-\alpha \gamma^{2} \cos ^{2} \theta\right) .
$$

DeSimone and Dolzmann [8], showed that any uniaxial macroscopic elongation $\lambda \leq \gamma$ can be realized by a textured pattern of deformations which are all uniaxial elongations by $\gamma$. Taking any such pattern, its energy averaged across the whole material will simply be the above expression with $\cos (\theta)$ replaced by its average, $1 / 3$. This average is independent of the relative length scales of the variation in $\hat{\mathbf{n}}_{0}$ and $\underline{\underline{\gamma}}$. We expect that in practice the material achieves a lower energy than this upper bound by correlating the stretch axes and preferred directions. Minimizing this energy with respect to $\gamma$ gives a minimum of $F=$ $\frac{3}{2} \mu(1+2 \alpha r / 3)^{1 / 3}$, at an extension of $\gamma^{3}=r /(1+$ $2 \alpha r / 3)$. The DeSimone and Dolzmann result therefore lets us place a bound on the energy of imposing a macroscopic stretch by $\lambda$ as

$$
\frac{2 F^{r}(\lambda)}{\mu} \leq \begin{cases}3\left(1+\frac{2 \alpha r}{3}\right)^{1 / 3} & \lambda^{3} \leq r /(1+2 \alpha r / 3) \\ r^{1 / 3}\left(\frac{2}{\lambda}+\left(\frac{1}{r}+\frac{2 \alpha}{3}\right) \lambda^{2}\right) & \lambda^{3} \geq r /(1+2 \alpha r / 3) .\end{cases}
$$

We find a Sachs lower bound on the energy by assuming each domain is subject to the same stress. This approach does not require that the deformation field be compatible, so it is a lower bound on the energy. Therefore, at zero stress every domain undergoes its preferred spontaneous deformation-a uniaxial stretch along its preferred director. Since the sample is macroscopically isotropic, this leads to no macroscopic deformation, but the energy density is minimized at every point as $F=3 \mu / 2$. Computing the lower bound at finite extension can be done simply numerically by minimizing $F\left(\underline{\underline{\gamma}}, \hat{\mathbf{n}}, \hat{\mathbf{n}}_{0}\right)-\sigma \gamma_{x x}$ over all $\underline{\underline{\gamma}}$ and $\hat{\mathbf{n}}$ at fixed $\sigma$ for a given domain $\left(\hat{\mathbf{n}}_{0}\right)$ to find the optimal deformation $\underline{\underline{\gamma}}_{m}$ and director orientation $\hat{\mathbf{n}}_{m}$ of the domain at the stress $\sigma$. The energy and extension of the whole sample are then found by averaging $F\left(\underline{\underline{\gamma_{m}}}, \hat{\mathbf{n}}_{m}, \hat{\mathbf{n}}_{0}\right)$ and 
$\left(\gamma_{m}\right)_{x x}$ over all domain orientations. A simpler analytic lower bound is provided by the exact ideal relaxed energy [above Eq. (3)] since the extra nonideal term is always positive.

The bounds on the energy are shown in Fig. 3. They require that the elastomer can be stretched by $\lambda^{3}=r /(1+$ $2 \alpha r / 3) \sim r$ at a maximum energetic cost of $\alpha \mu r / 3$. Since $F \sim \sigma(\lambda-1)$ deformations up to this point must occur at stresses $\sigma \sim \alpha \mu r / 3\left(r^{1 / 3}-1\right)$. These are proportional to $\alpha$, so these extensions will take place at very low stress, and in this regime the elastomer will undergo textured deformations. At strains higher than $\lambda^{3}=r /(1+2 \alpha r / 3)$ the stress will rise rapidly in the same way as the ideal case, and the director will be uniform. The derivative of the Sachs lower energy bound provides an estimate of the stress-strain curve that is also compared with a real elastomer in Fig. 2. The fit is good, although the soft stressplateau is flatter in the data. Although derivatives of energy bounds provide estimates of the stress, they are not stress bounds, which will be discussed in a forthcoming publication.

We have established three striking behaviors in isotropic genesis polydomains: domain structures driven by mechanical compatibility, extremely low nonideality, and macroscopic supersoft elasticity. We do not expect nematic genesis polydomains to exhibit any of these three behaviors. First, they are cross-linked in the nematic state, so their domain structure will be that of a nematic liquid, namely, a disclination texture. Second, their cross-linking state is not at all isotropic-it can distinguish the direction of the nematic director $\hat{\mathbf{n}}_{0}$ so nonideality will probably be similar to that found in monodomains, in which $\alpha \sim 0.1$. Either of these factors would be enough to destroy the third behavior-macroscopic supersoftness. This is clear in the case of elevated values of $\alpha$ since no deformations can take place on energy scales below $\alpha \mu$. However, even if $\alpha$ were zero, we would not expect macroscopic soft behavior because the deformation $\underline{\underline{\lambda}}(\mathbf{x})=\underline{\underline{\ell}}_{0}^{-1 / 2}(\mathbf{x})$ will not be mechanically compatible, so the material does not have a global isotropic reference state to generate softness. Recent

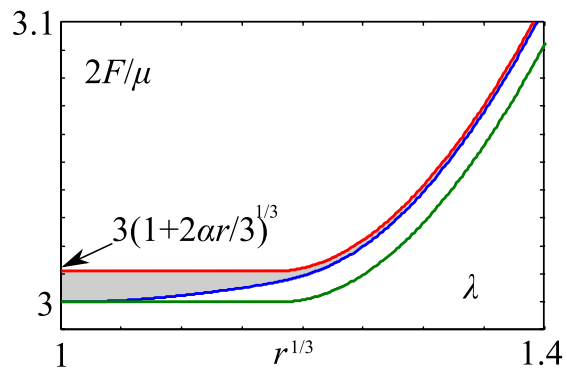

FIG. 3 (color online). Bounds on the free energy density of an isotropic genesis polydomain as a function of strain, plotted in units of $\mu / 2$ with $r=1.65$ and $\alpha=0.01$. Upper curve: Upper bound from a test strain field. Middle curve: Sachs lower bound. Lower curve: Ideal result, lower bound. The permissible region between the bounds is shaded gray. results confirm that nematic genesis polydomains do not show any soft stress plateau at all [9]. If a nematic genesis polydomain is heated to the "isotropic," it will be unable to undergo the energy minimizing contraction $\underline{\ell}_{0}^{-1 / 2}$ everywhere, so the material will be internally stressed. This may well be visible between cross-polars and lead to elevated nematic-isotropic transition temperatures.

Finally, we note that our conclusions about isotropic genesis elastomers probably generalize to other types of liquid crystal order, including $\operatorname{Sm} A$ and $\operatorname{Sm} C$, provided the cross-linking occurs in the fully isotropic state since the spontaneous deformations associated with the isotropic$\mathrm{Sm}$ transition are very similar to those associated with the isotropic-nematic transition. We also expect our methods to work and our conclusions to apply to nonideal isotropic genesis models with any other form of nonideality, relying only on the nonideal term being small and introducing a preferred direction.

We gratefully acknowledge the financial support of the Powell Foundation (Biggins), the Sims fund (Biggins), and the EPSRC (Bhattacharya and Warner). We thank K. Urayama for permission to reproduce the data in Fig. 2.

[1] M. Warner and E. M. Terentjev, Liquid Crystal Elastomers (Oxford University Press, Oxford, 2007).

[2] M. Warner, P. Bladon, and E. M. Terentjev, J. Phys. II (France) 4, 93 (1994).

[3] P. D. Olmsted, J. Phys. II (France) 4, 2215 (1994).

[4] L. Golubovic and T. Lubensky, Phys. Rev. Lett. 63, 1082 (1989).

[5] J. Kupfer and H. Finkelmann, Macromol. Chem. Phys. 195, 1353 (1994).

[6] H. Finkelmann, I. Kundler, E. M. Terentjev, and M. Warner, J. Phys. II 7, 1059 (1997).

[7] S. Clarke, E. Terentjev, I. Kundler, and H. Finkelmann, Macromolecules 31, 4862 (1998).

[8] A. DeSimone and G. Dolzmann, Arch. Ration. Mech. Anal. 161, 181 (2002).

[9] K. Urayama, E. Kohmon, M. Kojima, and T. Takigawa, Macromolecules 42, 4084 (2009).

[10] S. V. Fridrikh and E. M. Terentjev, Phys. Rev. Lett. 79, 4661 (1997).

[11] P. Bladon, E. M. Terentjev, and M. Warner, Phys. Rev. E 47, R3838 (1993).

[12] G. C. Verwey and M. Warner, Macromolecules 30, 4189 (1997).

[13] J. S. Biggins, E. M. Terentjev, and M. Warner, Phys. Rev. E 78, 041704 (2008).

[14] S. V. Fridrikh and E. M. Terentjev, Phys. Rev. E 60, 1847 (1999).

[15] S. M. Clarke, A. Hotta, A. R. Tajbakhsh, and E. M. Terentjev, Phys. Rev. E 64, 061702 (2001).

[16] G. C. Verwey and M. Warner, Macromolecules 30, 4196 (1997).

[17] K. Bhattacharya, Microstructure of Martensite (Oxford University Press, Oxford, 2003).

[18] E. Fried and S. Sellers, J. Chem. Phys. 124, 024908 (2006). 